\title{
The stellar population and metallicity distribution of the Sombrero galaxy
}

\author{
Jeremy Mould \\ School of Physics, University of Melbourne, Vic 3010, Australia \\ Lee Spitler \\ Centre for Astrophysics 83 Supercomputing, Swinburne University, Hawthorn, Vic 3122, \\ Australia
}

\begin{abstract}
Hubble Space Telescope studies of the resolved stellar population of elliptical galaxies have shown that the galaxies form by steady accretion of gas which is all the while forming stars and evolving chemically to a metallicity distribution that is as high as solar composition in the most massive objects that have been analyzed, and much lower for low mass ellipticals. In this paper we study for the first time the stellar content of an early type spiral galaxy, the massive disk galaxy, the Sombrero, NGC 4594. We consider whether the metallicity distribution function (MDF) in the observed field matches that of elliptical galaxies of some luminosity, and what these data imply for the accretion and enrichment model that can be fitted to the MDF. The MDF of NGC 4594 is similar to that of the elliptical galaxy of similar luminosity, NGC 5128. The field we are probing is a combination of the galaxy's bulge and halo.
\end{abstract}

Subject headings: galaxies: abundances - galaxies: photometry - galaxies: formation

\section{Introduction}

Deep imaging of nearby elliptical galaxies has in recent years opened up their stellar populations for analysis of the distribution of metallicity. Harris et al. (2007) has shown that the metallicity distribution is measurable in a range of galaxies from the dwarf spheroidals to giant ellipticals such as NGC 5128, and that the the metallicity distribution functions (MDF) are sequenced in luminosity, such that Draco is the most metal poor in mean Z, L* galaxies are intermediate, and NGC 5128 the most metal rich. In massive galaxies the MDF 
in metallicity peaks below solar $\mathrm{Z}$ and has an extended tail to very low metallicities. Lower mass galaxies show a Gaussian MDF at lower metallicities, see e.g. Lianou et al. (2010). The MDF constrains models of elliptical galaxy formation, including monolithic collapse models, hierarchical merging models, and merger scenarios over cosmic time, see e.g. Ikuta (2007).

Nearby edge-on galaxies present a unique opportunity to learn about the formation history of the bulge, thick disk, and halo of galaxies. Seth, Dalcanton, \& de Jong (2005) and Mould (2005) have examined HST imaging of two samples of late type edge-on galaxies and found thick disks without metallicity gradients and without asymptotic giant branch stars above the red giant branch tip. These observations rule out models of slow collapse and of young disk heating as thick disk formation mechanisms. Models that would be favored include those of Brook et al (2004) whose chemo-dynamical cosmological simulations identify thick disk formation with a $\mathrm{z}>2$ epoch of chaotic merging of multiple peer disks of mixed gas and stars.

Bulge dominated disk galaxies can be studied in the edge-on case in the same way. A superb example is the Sa galaxy NGC 4594. We present such a study in this paper and compare it with the Milky Way analog, NGC 891, (Rejkuba et al. 2009; Ibata et al. 2009), as well as with its elliptical siblings. There is no evidence of a thick disk in the perpendicular profiles of Burkhead (1986), whose scale height is approximately 400 pc. So we concentrate on the bulge and halo. The Sombrero galaxy is the first early type spiral to be so studied, although, in a not-so-edge-on case, we note the work of Richardson et al. (2009) on M31, which is limited to the outer halo. Mouhcine et al. (2005) has studied the disk galaxies NGC $55,247,253,300,3031,4244,4258$, and 4945. They find that the MDFs are dominated by metal-rich populations, with a tail extending toward the metal poor end and that, to first order, the MDFs are similar to what is predicted by simple, single-component model of chemical evolution with the effective yields increasing with galaxy luminosity.

\section{Photometry}

The HST archive contains six undithered F606W WFPC2 exposures of a field in the Sombrero galaxy and six exposures in F814W, amounting to 11,700 seconds in each filter. These were obtained as part of the medium deep survey of the original HST Key Projects, GO program 5369. The field is 6.8 effective radii from the center of the galaxy and is located

as depicted in Figure 1. According to Young (1976) 90\% of the luminosity of an $r$ to the quarter law spherical bulge is contained within this radius, and so we can expect a significant fraction of the stars in this field to be halo stars. 
The frames were combined with IRAF and processed with DAOPHOT (Stetson 1987). A sample of the field is shown in Figure 2, allowing an impression of the level of crowding. Point spread functions were borrowed from the extragalactic distance scale Key Project (Kennicutt, Mould, \& Freedman 1995) and fitted to the images with ALLSTAR. The data underwent a second cycle with stars found in the residual image. The ALLSTAR files were then combined with DAOMASTER. The number of stars detected on the four chips was $3647,10,030,10,158$ and 11,852, where chip 1 is the planetary camera. Nine bright stars were used to determine aperture corrections to an accuracy of $0.01 \mathrm{mag}$. The calibration of Dolphin (2000) was employed to transform the color magnitude diagram (CMD) to the VI system. There are spatial density gradients across the WFPC2 field in the point sources detected of 10-20\%. Both the PC and WF chips are used. The stellar density increases in the field in the direction towards the center of the galaxy.

The CMD is shown in Figure 3. Stars have been excluded if their color uncertainties exceed $0.5 \mathrm{mag}$. The CMD shows the tip of the red giant branch (TRGB) of NGC 4594. The TRGB is at $\mathrm{I}=26.2 \pm 0.2 \mathrm{mag}$, corresponding to $(m-M)_{0}=30.15 \pm 0.2 \mathrm{mag}$ (see Mould \& Sakai (2008)). The population is an old one.

NGC 4594 is at a distance of $29.95 \pm 0.18 \mathrm{mag}$ according to surface brightness fluctuation measurements by Tonrv et al. (2001). This is the distance we adopt. The reddening is $\mathrm{E}(\mathrm{V}-\mathrm{I})=0.071$ from Schlegel et al. (1998). From the distribution of giant branch colors a magnitude or so fainter than $\mathrm{M}_{I}=-4.0$, it is thus possible to measure the MDF, following Harris et al. (2007), who provide template giant branches for this purpose. The MDF is obtained by interpolation directly in color-magnitude space and is shown in Figure 4 . The isochrone age was 12 Gyrs and the isochrone spacing was 0.15 dex in $[\mathrm{Fe} / \mathrm{H}]$.

Eight hundred artificial stars with $\mathrm{V}-\mathrm{I}=1.75 \mathrm{mag}$ and $26<\mathrm{I}<28$ were added to the F606W and F814W frames, and the measurement process was repeated. The average color error was $0.42 \mathrm{mag}$ with little dependence on magnitude. The average I magnitude error was $0.44 \mathrm{mag}$. The color bias due to blending was measured at $-0.02 \pm 0.03$, which is negligible. The process was repeated for $\mathrm{V}-\mathrm{I}=3$, and the color error rose to 0.54 mag. Since the sensitivity of color to metallicity increases in the red, this increase is not an issue for the MDF. Incompleteness affects the MDF at the metal rich end. However, this effect is modest: a factor of two incompleteness in the solar metallicity bin in Figure 4. But a small high metallicity tail with $[\mathrm{Fe} / \mathrm{H}]>0$ may have been erased by incompleteness. Figure 5 results from photometry of a further 2400 artificial stars and shows completeness as a function of I magnitude. 


\section{Chemical evolution}

Since the time of Pagel \& Patchett (1975) the MDF has been used to constrain the chemical evolution of galaxies, starting with the Milky Way. With deviations from the closed box chemical enrichment assumption it is possible to fit a metal rich distribution like that of the solar neighborhood with $d A / d S \rightarrow \alpha$, where $A$ is the mass of accreted gas, $S$ is the mass of stars made, and $\alpha$ is the fraction of low mass long-lived stars formed in a generation of star formation. Hartwick (1976) showed that a metal poor MDF, such as that of Galactic halo globular clusters, could be formed with gas loss from the system, all with standard values of the yield, $p$, the fraction of heavy elements, $Z$ created in a generation of star formation and return to the interstellar medium. His equation (7) can be differentiated to yield:

$$
\frac{d S}{d Z} \propto e^{-(\alpha+c) Z / p}
$$

where $c$ is the ratio of gas lost to stars formed, when star formation is complete. The resulting MDFs for different values of $c$ are shown in Figure 6.

We also consider a somewhat more general case in which the MDF can be used to constrain $A(t)$. If $\mu$ is the gas fraction at time $t$, we have equation (34) of Pagel \& Patchett (1975) (mass conservation).

$$
\mu(t)=1-\alpha S(t)+A(t)
$$

If $Z^{\prime}$ is the $Z$ of the accreted gas, heavy element accounting (equation (6) of Pagel \& Patchett $(1975))$ gives us:

$$
\mu d Z=\alpha p d S-\left(Z-Z^{\prime}\right) d A
$$

Then the MDF can be written as:

$$
\frac{d S}{d Z}=\frac{\mu}{\alpha p-\left(Z-Z^{\prime}\right) d A / d S}
$$

So, given the star formation history, $\mathrm{S}(\mathrm{t})$, the accretion history, $\mathrm{A}(\mathrm{t})$, and the SchmidtKennicutt law, dS/dt $\propto \mu^{n}, 1<n<2$, one can predict the MDF and compare with observations. An alternative way to proceed is to use $\mathrm{Z}(\mathrm{t})$ as a proxy for time and parameterize the gas depletion history. 


$$
\mu=e^{-Z /(p x)}
$$

where $x$ is a free parameter controlling the rate of star formation decline. We can then use the MDF directly to calculate the accretion rate.

$$
\left(Z-Z^{\prime}\right) \frac{d A}{d Z}=\alpha p \frac{d S}{d Z}-\mu
$$

Figure 7 shows the solution for the Sombrero's MDF. The accreted gas metallicity was taken to be $\mathrm{Z}^{\prime}=0.0005$ and $\mathrm{x}$ was put equal to 0.5 . Values of the yield and $\alpha$ were taken from Pagel \& Patchett (1975). The interesting results are that there is an early burst of accretion and that $\mathrm{dZ} / \mathrm{dt}$ is fairly steady, consistent with the assumption that $\mathrm{Z}$ is a good proxy for time. If $\mathrm{dZ} / \mathrm{dt}$ is $\sim 0.02 / 13 \mathrm{Gyr}$, the duration of the initial accretion burst is $\sim 0.17$ Gyr. Other choices of parameters yield similar results, although $\mathrm{dZ} / \mathrm{dt}$ becomes monotonic decreasing, if the gas depletion history is rapid.

\section{Discussion}

Our measurement of the Sombrero's MDF is, however, severely affected by photometric errors. As discussed in $\S 3$, these range from 0.42 to 0.54 mag in color, moving from blue to red on the giant branch. This corresponds to $-1,+0.3$ dex at $[\mathrm{Fe} / \mathrm{H}]=-1$ and \pm 0.2 dex at $[\mathrm{Fe} / \mathrm{H}]=-0.4$. To examine this, we ran a simulation with 800 artificial stars with $\mathrm{I}=26.4$ and $\mathrm{V}-\mathrm{I}=1.75$. That is a delta function in metallicity with $[\mathrm{Fe} / \mathrm{H}]=-0.875$. The simulated MDF (generated in exactly the same way as for the real data) showed a strong low metallicity wing but fewer solar metallicity stars than Figure 4 . We therefore ran the metallicity delta function through our $\mathrm{dA} / \mathrm{dZ}$ calculation. The result is very similar to Figure 7 with a similar early accretion peak.

It is interesting to note that the peak of the MDF in Figure 4 coincides with the metalrich peak of the globular cluster MDF of Harris et al. (2010).

Harris et al. (2007) have shown that the MDFs of the elliptical galaxies they have studied (see their figure 15) form a sequence in increasing luminosity and increasing mean metallicity. With a luminosity $\mathrm{M}_{B}=-21.2$ and modal metallicity $[\mathrm{Fe} / \mathrm{H}]=-0.5$, NGC 4594 is similar to NGC 5128 with $\mathrm{M}_{B}=-20.6$ and modal metallicity -0.45 (de Vaucouleurs et al. 1991), (Harris et al. 2009). The bulge dominated Sombrero galaxy is thus similar chemically to its equivalent elliptical galaxy and much more metal rich than the Milky Way analog NGC

891 (Rejkuba et al. 2009) $([\mathrm{Fe} / \mathrm{H}] \approx-1.0)$. This suggests that similar processes are occurring 
to determine the chemical composition of early type spiral galaxy halos and elliptical galaxies.

Our models of chemical evolution directed at the MDFs of galaxies are consistent with the idea that accretion history and gas loss may be key determinants. Low mass galaxies are unable to retain their gas and display low mean metallicity. High mass galaxies accrete substantial gas early in their evolution and process that gas to a high mean metallicity. The mass sequence of Harris et al. (2007)'s MDFs from the Draco dwarf, through the Leo ellipticals to NGC 5128 may thus be explained through the agency of gas loss and accretion.

We have glibly spoken of the field we have observed as characteristic of NGC 4594. A full study of the stellar population of the Sombrero would search for chemical gradients in the galaxy, characteristic of chemical enrichment during collapse and seek distinctions between the bulge, disk, and globular cluster MDFs. We leave this for further work.

\section{Conclusions}

The MDF of a field 6.8 effective radii from the center of the Sa galaxy NGC 4594 has a modal metallicity of $[\mathrm{Fe} / \mathrm{H}]=-0.5$, similar to that of NGC 5128, an elliptical galaxy of similar luminosity. This is the first early type disk galaxy to have its MDF measured.

Such a metal rich MDF can be achieved in accreting chemical evolution models with an initial burst of gas accretion lasting of order 0.17 Gyrs. This is the time elapsed between redshifts as high as 14 and 10. Alternatively, a loss of two thirds of the gas from this field during star formation, say, on to the disk could have yielded the observed MDF.

Higher signal to noise CMDs of the Sombrero over multiple fields will help to unravel the star formation and chemical enrichment history of galaxy bulges.

We thank the referee for comments which have improved the paper. This work is based on data obtained with the NASA/ESA Hubble Space Telescope. In addition to DAOPHOT, this research has made use of IRAF. IRAF is distributed by NOAO which is operated by AURA for NSF.

\section{REFERENCES}

Brook, C. et al. 2004, ApJ, 612, 894

Burkhead, M., AJ, 91, 777

Da Costa, G. \& Armandroff, T. 1990, AJ, 100, 162 
de Vaucouleurs, G. et al. 1991,Third Reference Catalog of Bright Galaxies, Springer-Verlag: New York

Dolphin, A. 2000,PASP, 112, 1397

Harris, W. et al. 2007, ApJ, 666, 903

Harris, G., Rejkuba, M., \& Harris, W. 2009, astro-ph 0911.3180

Harris, W. et al. 2010, MNRAS, 401, 1965

Hartwick, D. 1976, ApJ, 209, 418

Ibata, R. et al. 2009, MNRAS, 395, 126

Ikuta, C. 2007, A\&A, 472, 77

Kennicutt, R., Mould, J. \& Freedman, W. 1995,AJ, 110, 1476

Lianou, S. et al. 2010, astroph 1003.0861

Mouhcine, M. et al. 2005, ApJ, 633, 828

Mould, J. 2005, AJ, 129, 698

Mould, J. \& Sakai, S. (2008), Astrophys. Lett., 686, L75

Pagel, B. \& Patchett, B. 1975, MNRAS, 172, 13

Rejkuba, M. et al. 2009, MNRAS, 396, 1231

Richardson, J. et al. 2009, MNRAS, 396, 1842

Schlegel, D. et al. 1998, ApJ, 500, 525

Stetson, P. 1987, PASP, 99, 191

Tonry, J. et al. 2001, ApJ, 546, 681

Young, P. 1976,AJ, 81, 807 


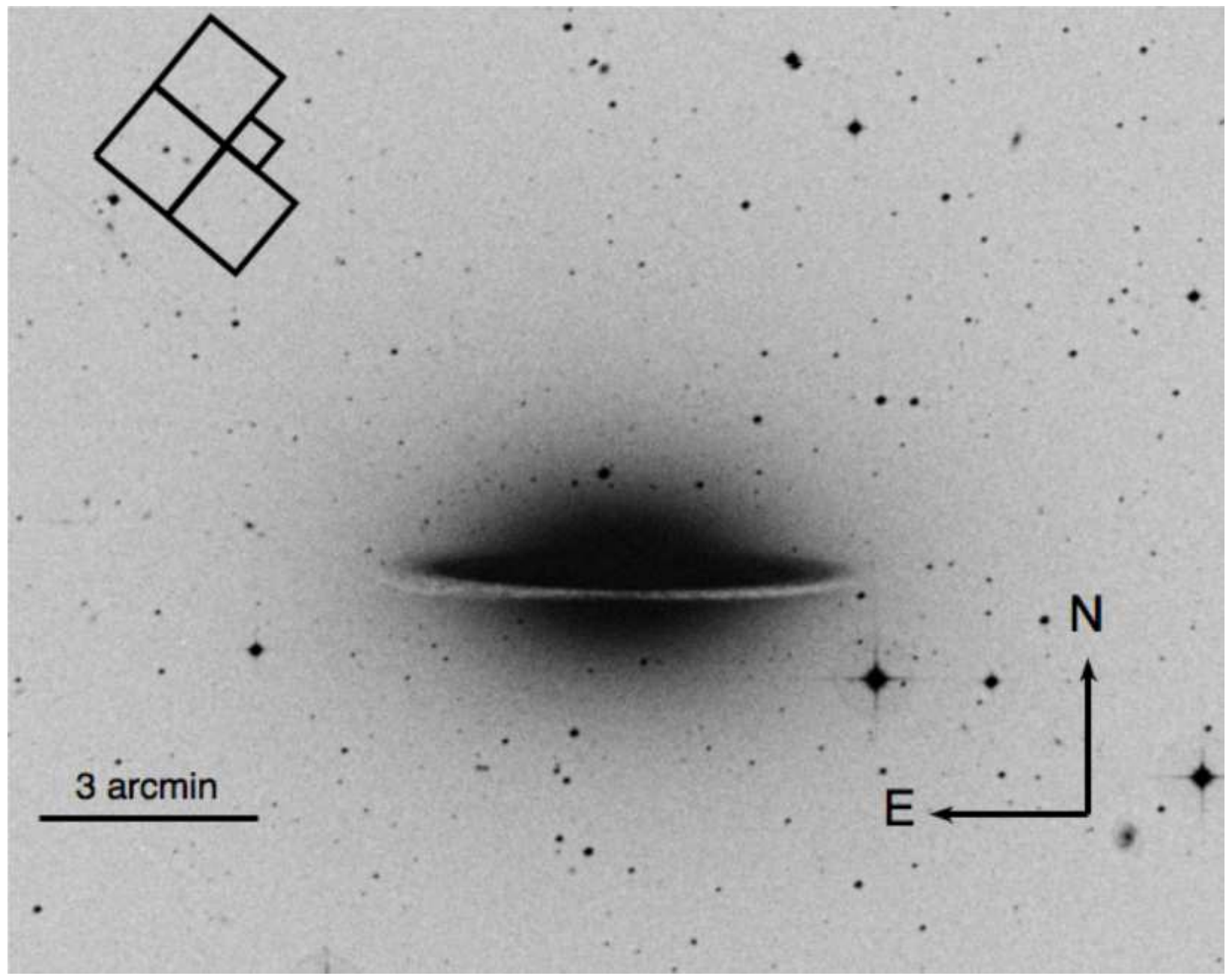

Fig. 1. - The field of NGC 4594 with the WFPC2 footprint shown. 


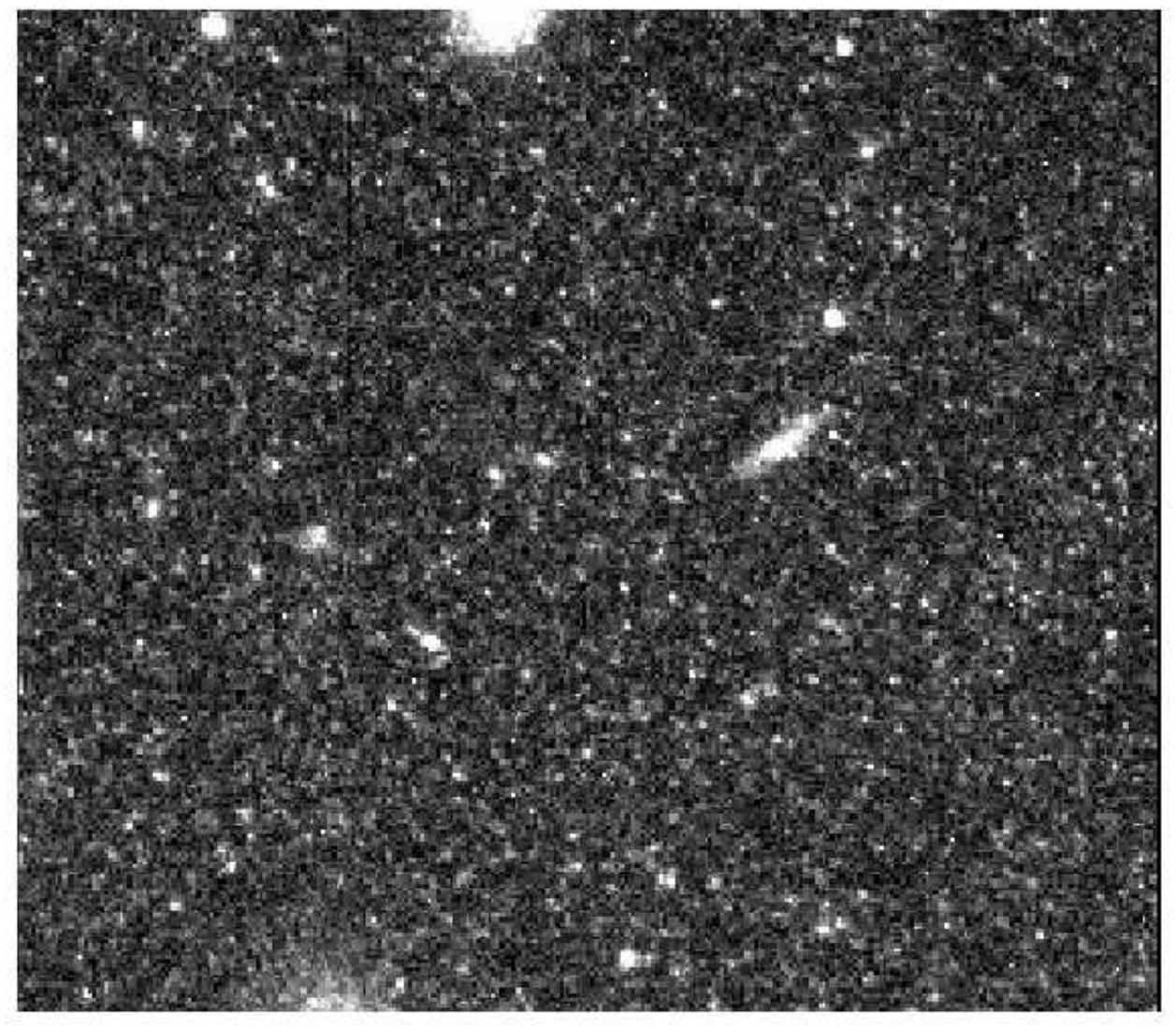

Fig. 2.- An enlargement of a $300 \times 300$ pixel area in the N4594 field from the center of chip 2 . 


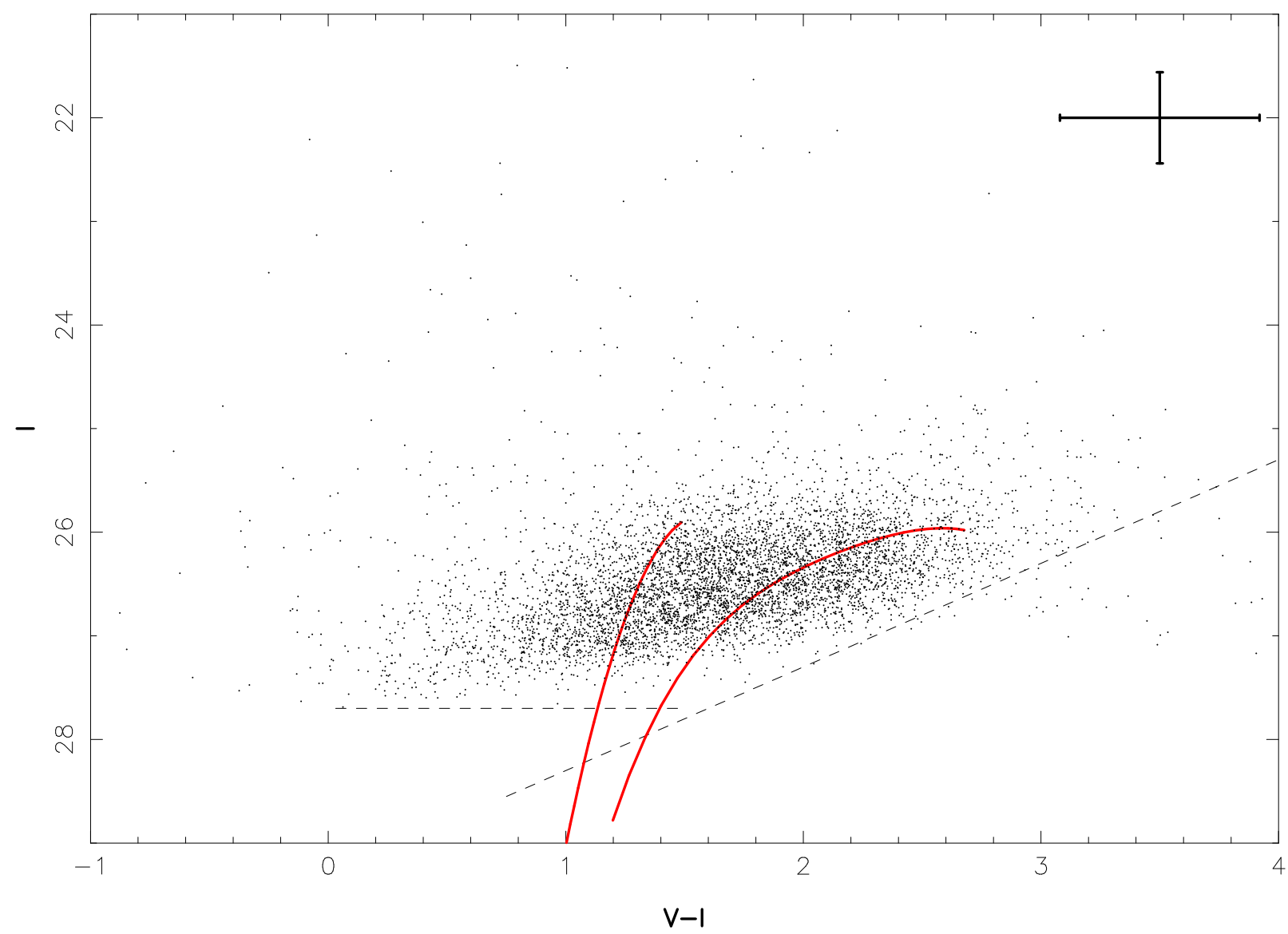

Fig. 3.- Color magnitude diagram of NGC 4594. The solid curves are the giant branches of M15 and 47 Tuc from Da Costa \& Armandroff (1990) moved to the distance and reddening of the galaxy. The dashed lines are the $50 \%$ completeness lines $\mathrm{V}=29.3 \mathrm{mag}$ and $\mathrm{I}=27.7$ mag. M15 has $[\mathrm{Fe} / \mathrm{H}]=-2.17$ and 47 Tuc has $[\mathrm{Fe} / \mathrm{H}]=-0.71$. 


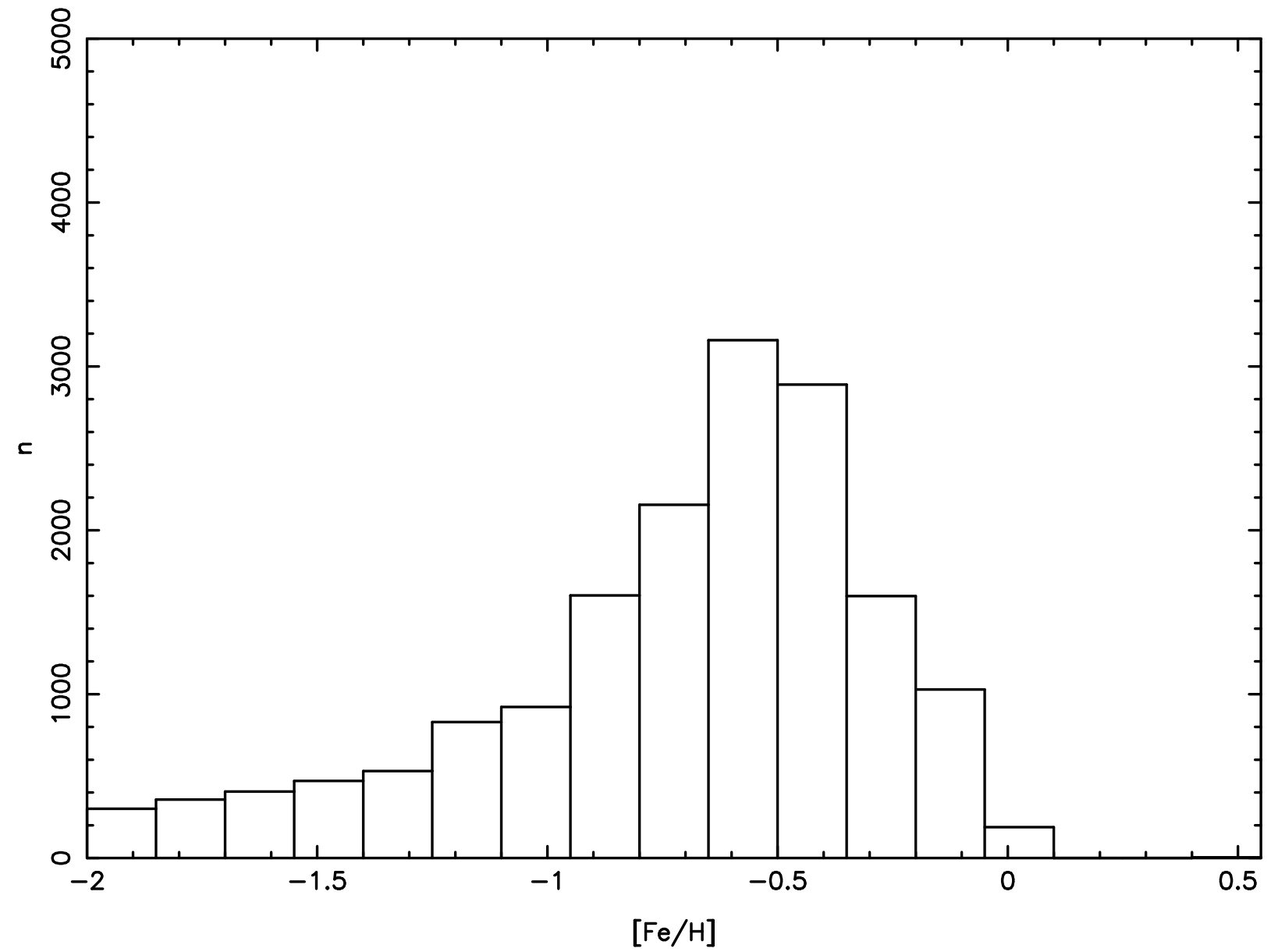

Fig. 4. - Metallicity distribution of the detected red giants in NGC 4594. 


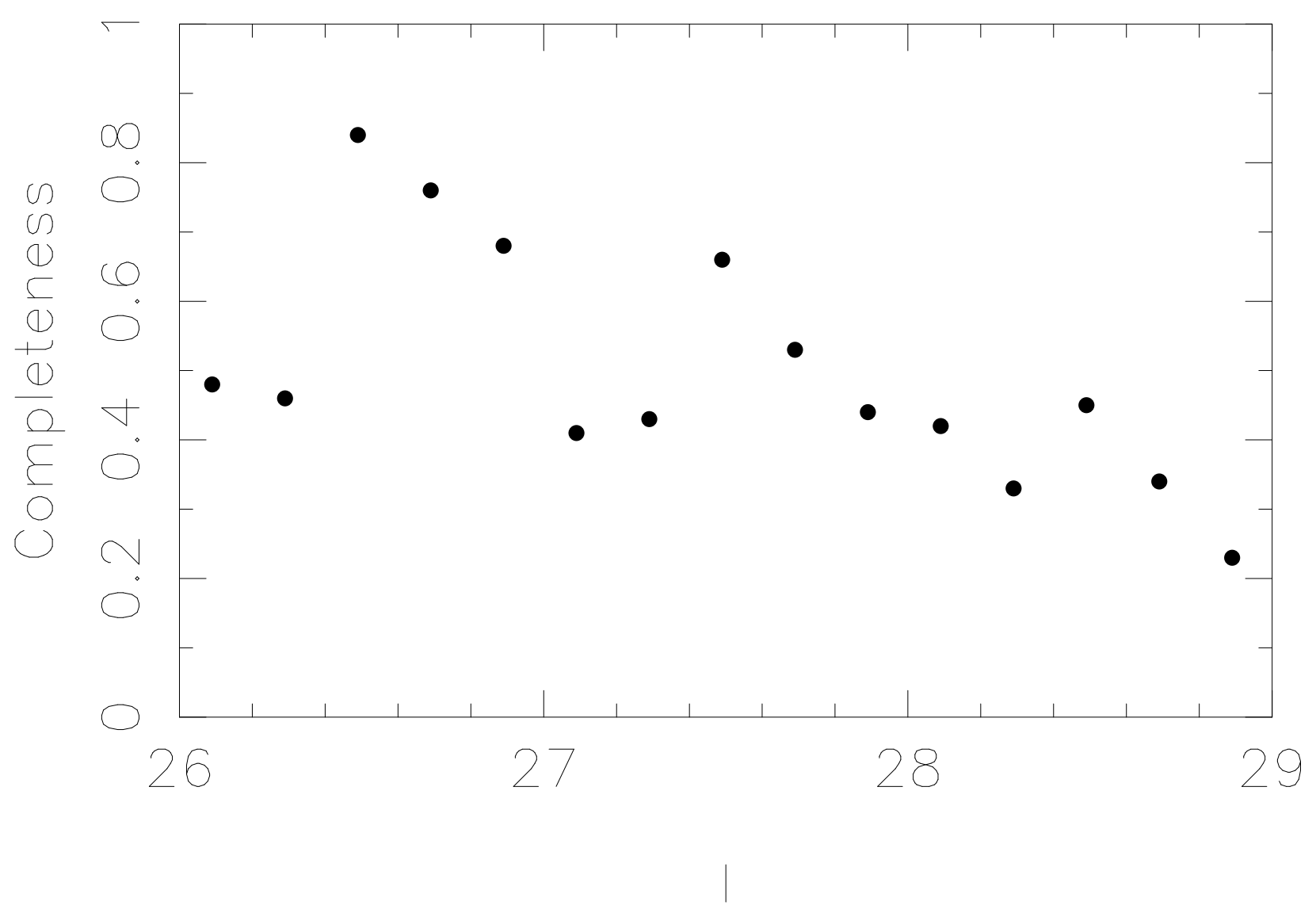

Fig. 5.- Photometric completeness as a function of I magnitude. The $50 \%$ completeness level shown in Figure 3 is at $\mathrm{I}=27.7 \pm 0.3 \mathrm{mag}$. 


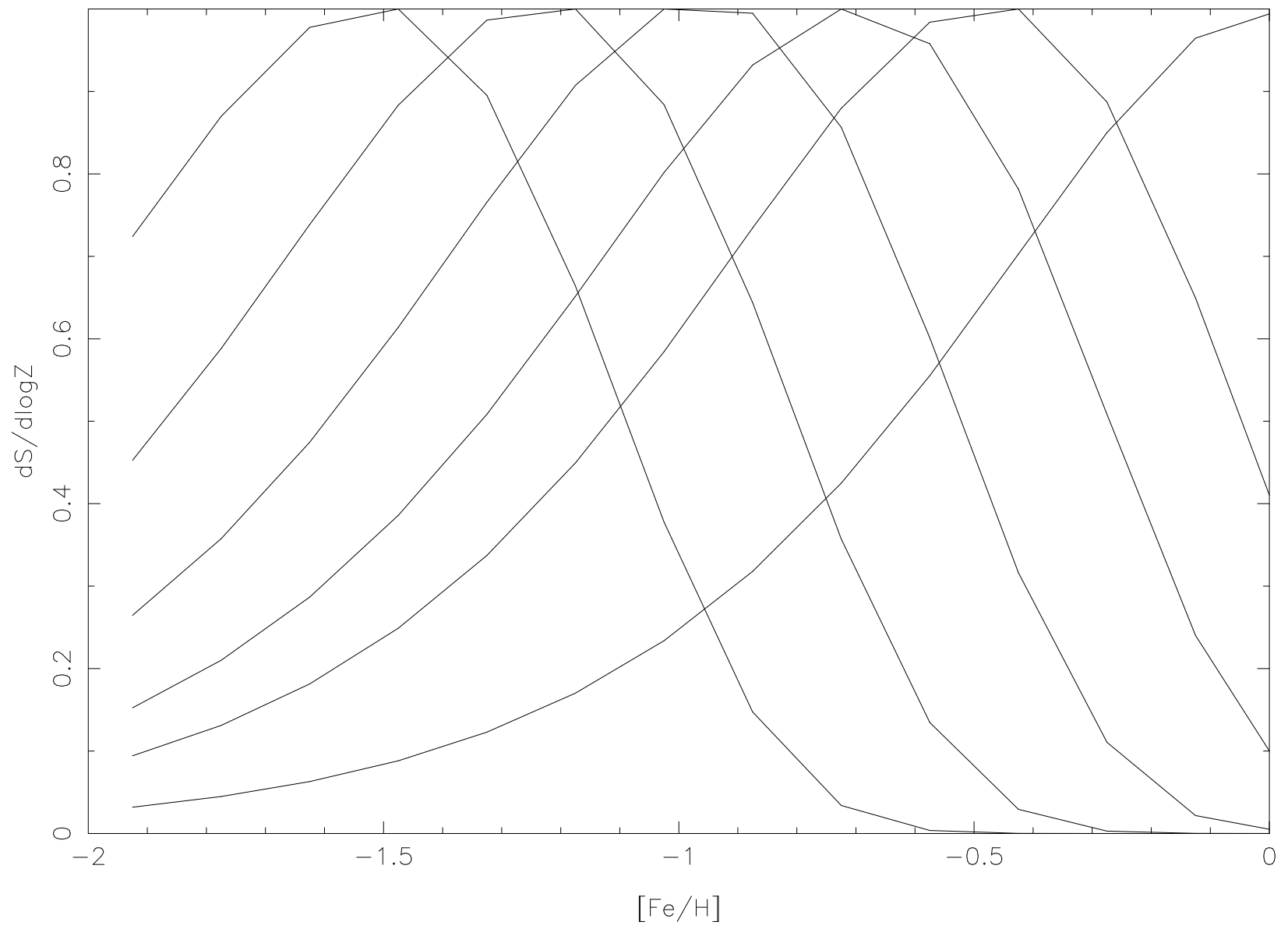

Fig. 6.- Metallicity distribution in the simple model of chemical evolution with gas loss. The ratio of gas lost to stars formed takes the values for distributions from left to right of $32,16,8,4,2,0$. 

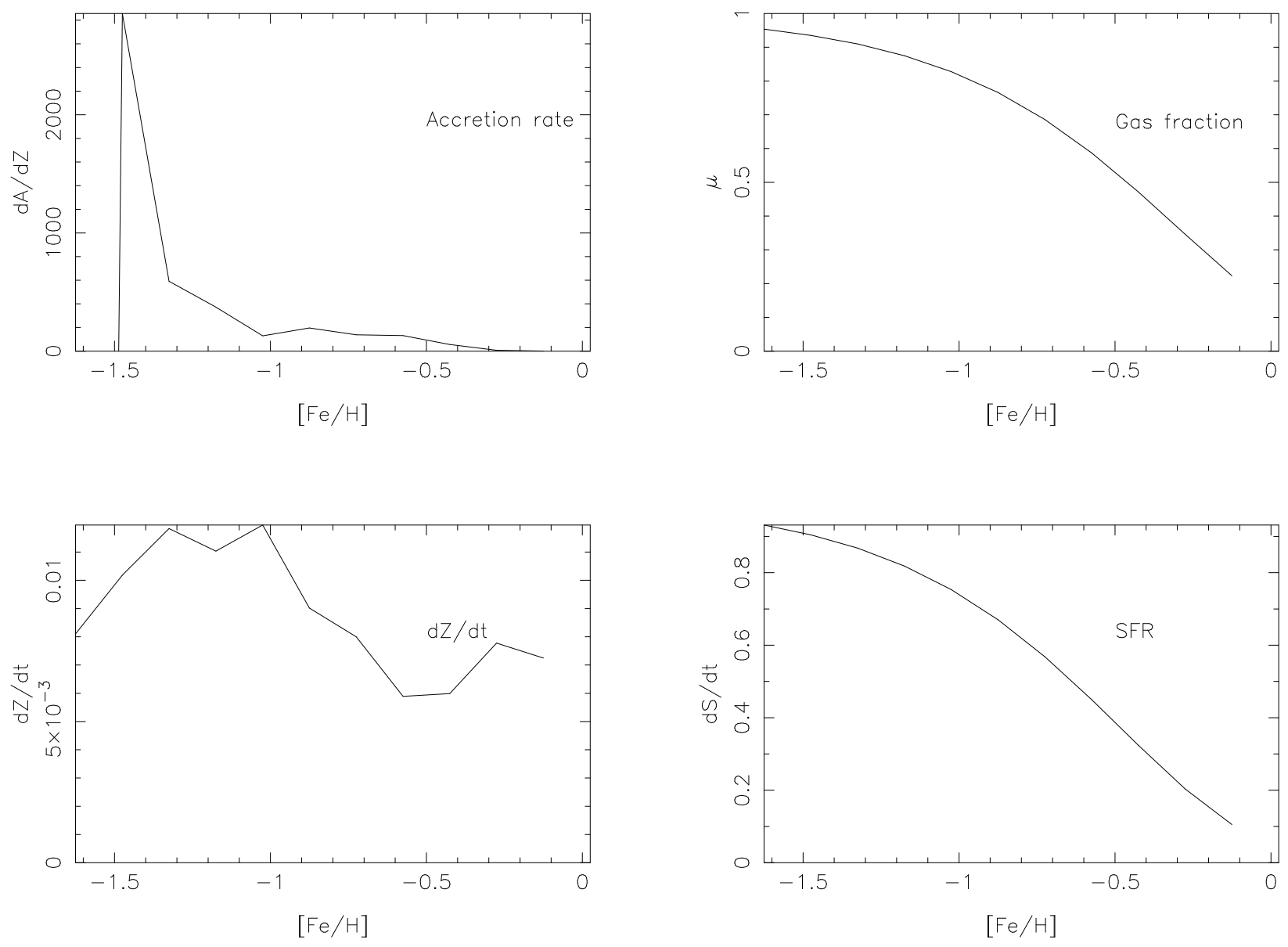

Fig. 7.- The accretion rate as a function of metallicity (top left) in NGC 4594. The independent variable, $[\mathrm{Fe} / \mathrm{H}]=\log Z / Z_{\odot}$, can be considered to be a proxy for time. The assumed gas depletion rate is depicted in the top right. The chemical enrichment rate (arbitrary units) is at the bottom left, and the star formation rate (arbitrary units) at the bottom right. 